\title{
THE EFFECTS OF LOW-ENERGY SCATTERING ON POSITRON IMPLANTATION
}

\author{
K. A. Ritley \\ Department of Physics and Materials Research Laboratory \\ University of Illinois at Urbana-Champaign \\ 1110 W. Green Street, Urbana, Il 61801 \\ K. G. Lynn, V. Ghosh, and D. O. Welch \\ Department of Physics and Department of Applied Science \\ Brookhaven National Laboratory, Upton, New York 11973
}

BNL -48129

DE93 005537

\section{ABSTRACT}

Existing Monte-Carlo models are capable of aimulating the behavior of positrons incident at $\mathrm{keV}$ energies, then following the energy loes process to aribitrary final kinetic energies of from $20 \mathrm{eV}$ to $100 \mathrm{eV}$. In the present work we describe a Monte-Carlo simulation of the final stages of positron thermalization in Al, from $25 \mathrm{eV}$ to thermal energies, via the mechanisms of conduction-electron and longitudinal acoustic phonon scattering. We show that the latter stages of thermalization can have important effects on the stopping profiles and mean depth. We describe a novel way to obtain information about positron energy loss by considering the time-evolution of a point-concentration (delta-function distribution) of positrons. We examine, for the first time in the context of a positron MonteCarlo calculation, the effects of a positive positron work function. Finally, we discuss some issues relating to the agreement of Monte-Carlo calculations with experimental data.

\section{INTRODUCTION}

The need for reliable Monte-Carlo models of positron implantation and stopping is clear. A slow positron incident on a solid rapidly thermalizes $\left(\sim 10^{-13} \mathrm{~s}\right)$ and then diffuses at thermal energies, trapping in defects and interacting with the surface. Annihilation with electrons does not occur until much longer times $\left(\sim 10^{-10} \mathrm{~s}\right)$. The simplicity of this two-step "stopping-and-diffusion" process, at least to first order, is fortuitous, as it facilitates the analysis of experimental data using a straightforward application of the diffusion equation. Although the diffusion aspect of this problem is far from trivial, it is readily attacked and a variety of solutions are available. Unfortunately the problem of determining the stopping profiles (i.e., the initial conditions for diffusion) is less amenable to analytical treatments. These conditions are usually characterized by the implantation (or stopping) profile $P(z)$, where $P(z) d z$ is the probability of locating a positron between $z$ and $z+d z$. Accurate experimental measurements of this quantity are difficult, and thus the experimentaliats have had to rely quite heavily on the results of computer-based Monte-Carlo calculations.

Monte-Carlo calculations are able to track the progress of a positron incident at high kinetic energies, usually in the keV range, and follow its trajectory through the material as it scatters both elastically and inelastically, until it has 
reached an arbitrary endpoint energy, at which point the positron is considered to be stopped. In the Monte-Carlo programs to date, that endpoint energy has been typically $20 \mathrm{eV}$ to $100 \mathrm{eV}$. At these energies, the inelastic process of positron-conduction-electron scattering will quickly deliver the positron to near-thermal energies, at which point phonon scattering becomes dominant and the diffusion process begins. Thus it has been argued that the final stages of thermalization, from $\sim 20 \mathrm{eV}$ to $\frac{3}{2} k_{B} T$, will have a negligible effect on the stopping profiles and other quantities generated by these programs.[1-4]

In the present work we have chosen to re-examine that assumption. Our motivation for this is essentially three-fold. First, although the time required for positrons to give up their final kinetic energy $\left(\sim 20 \mathrm{eV}\right.$ to $\left.\frac{3}{2} \mathrm{k}_{\mathrm{B}} \mathrm{T}\right)$ is extremely short, the physical effects of scattering depend on the mean scattering rates and on the mean free paths, which as we discuss below are not negligible. Second, there is a growing need for information about positrons incident at low energies on multilayers and buried interfaces. The length scales associated with these systems are relatively short and even small changes to the stopping profiles made by low-energy scattering could be important. Additionally, positron work function differences between adjoining materials may have some effect on positrons incident on such systems. Even with a relatively large binetic energy, the kinetic energy of the component of velocity normal to the surface may be of the order of the difference between work functions. Continuing the energy loss process to thermal energies allows us to examine possible work function effects. Third, these Monte-Carlo models have some utility as a straightforward and physically intuitive means for studying the crossover from positron stopping to positron diffusion. Although numerical solutions of the Boltzmann transport equation have been used to study such processes, it has been shown that stochastic approaches such as single-particle Monte-Carlo calculations are a direct and efficient way of numerically integrating the Boltzmann equation. $[5,6]$

This paper is organized in the following way: We triefly discuss in Sec. II the Monte-Carlo model and the scattering mechanisms it includes. We discuss in Sec. III a novel way to examine the energy loss process, by considering the time-evolution of a point concentration of positrons. In Sec. IV we discuss the effects of low-energy scattering on the stopping profiles and the mean depth, and in Sec. V we describe how a positive work function affects the stopping profiles. Finally, in Sec. VI, we discuss some important issues regarding the agreement of Monte-Carlo simulations with experimental data. Detailed results concerning backscattering and the energy distribution of the re-emitted positrons will appear in a forthcoming publication.[7]

\section{MODEL AND MECHANISMS}

In order to study the effects of low-energy inelastic scattering (in the $\sim 20 \mathrm{eV}$ to $\frac{3}{2} k_{B} \mathrm{~T}$ range), a single-particle Monte-Carlo model of the usual type was constructed.[1] The behavior of positrons contained within a semi-infinite sample is modelled by averaging a large number of single particle trajectories through the material. Using the notation of Ref. 14, a trajectory can be denoted by a 
series of states $\left(E_{n}, r_{n}, \Omega_{n}\right)$, where $E_{n}, r_{n}$ and $\Omega_{n}$ represent the energy, position vector and direction cosines of a particle after the $n^{\text {th }}$ scattering event. As a positron travels through the material, it scatters inelastically $\left(E_{n+1}<E_{n}\right)$, and its progress is followed until it is either backscattered out of the material $\left(r_{2}<0\right)$ or until its energy has fallen below the threshold energy for the simulation. Full details of the calculation of the scattering states are given in Ref. 7.

The initial conditions of the present work are taken to be the results of a Monte-Carlo calculation using BNLPOS. $[1,2]$ In that model an incident positron can be followed from a relatively high incident energy (keV) to an endpoint ezergy of $25 \mathrm{eV}$. An extensive complement of elastic and inelastic scattering processes is included; this is described in detail in Ref. 1 and 2.

The $25 \mathrm{eV}$ endpoint implantation profiles generated by BNLPOS are then fit to Mahkovian distributions (see Sec. 5), and these are used as input parameters for the present work. [8] The scattering mechanisms considered are conductionelectron scattering, using the free-electron-gas formalism developed by Oliva; and longitudinal acoustic phonon scattering, using the Debye-model formalism developed by Nieminen and Oliva. $[9,10]$ The combined mean free path per collision in the $\sim 20 \mathrm{eV}$ to $\frac{3}{2} \mathrm{k}_{\mathrm{B}} \mathrm{T}$ energy range is typically 30 Aor less in $\mathrm{Al}$. We do not consider plasmon scattering or elastic scattering. The former mechanism does not apply (for reasons of energy-momentum conservation) for positrons with energy below approximately $20 \mathrm{eV}$. The latter mechanism does not apply as the positron nears thermal energy and quantum effects become important; the usual cut-off for elastic scattering is typically twice the width of the lowest positron energy band.

We account for the surface in the following way. In the case of materials with positive positron work functions $\left(\phi_{+}>0\right)$, we consider the surface to be a simple energy step barrier of height $\phi_{+}$. Positrons incident on this barrier (from within the material) for which the kinetic energy $E_{\perp}$ of the component of velocity normal to the surface is less than the positron work function, $E_{\perp}<\phi_{+}$, are specularly reflected. For positrons with $E_{\perp}>\phi_{+}$, s small fraction is considered undergo quantum mechanical barrier reflection, according to the usual reflection coefficient for scattering off a square enerigy step barrier. For negative work function materials $\left(\phi_{+}<0\right)$, all positrons incident on the surface with the proper momentum are permitted to eacape freely. More complicated suface effects (such as trapping) are presently not included in this model.

\section{STOPPING AND DIFEUSION}

Our first step after constructing the Monte-Carlo model is to examine the process of positron stopping and the crossover from thermalization to diffusion. This is most easily accomplished by adopting for initial conditions a delta-function distribution of $25 \mathrm{eV}$ positrons, $P\left(t_{0}\right)=\delta\left(r-r_{0}\right)$, and then by tracking this distribution as the positrons scatter inelastically. The time-development of this distribution is depicted in Fig. 1, in which it it is seen that the initial distribution leads to Gaussian-like profiles which broaden over time. 


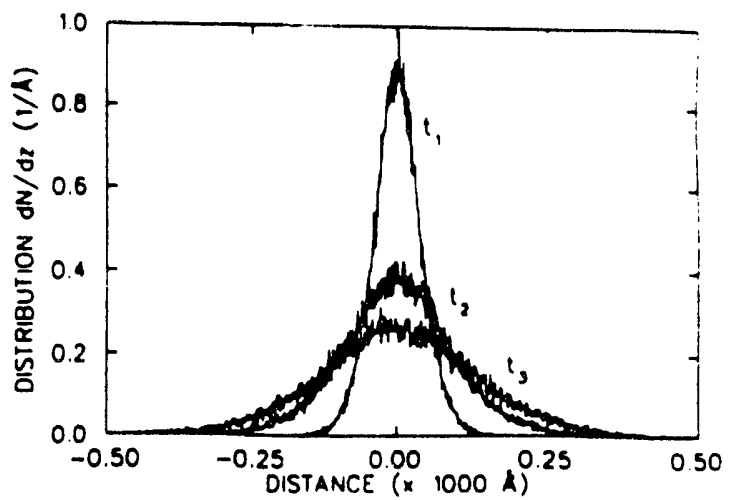

Pigure 1. The spreading of an initial point concentration of positrons, at $t=0.07$, 0.7 , and $2.2 \mathrm{ps}$.

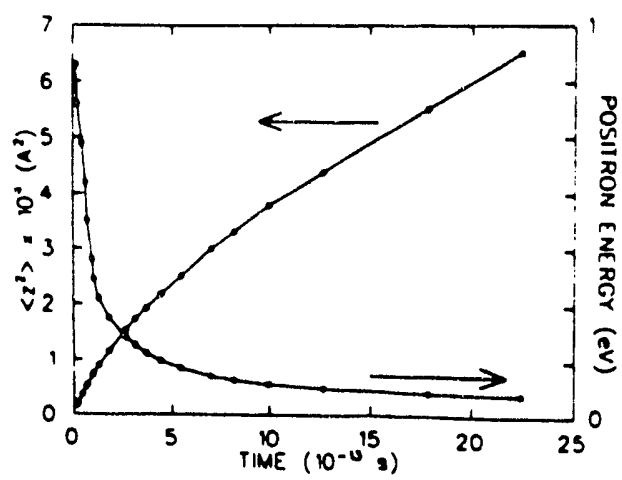

Figure a. The pocitron energy and the menn-aquare-displocement, both as functions of time, of the profles in Fig. 1.

The energy loss rate of the initial positron "cloud" appears in Fig. 2. This energy loss curve exhibits three distinct features: an abrupt initial descent in which conduction-electron scattering quickly delivers the positron to near-thermal energies (stopping domain); a somewhat longer interval in which the positrons continue to lose energy but in which the principal scattering mechanism shifts from conduction-electron scattering to phonon scattering (thermalization domain); and the final, phonon-scattering-dominated regime in which the energy loss is negligible (beginning of diffusion domain).

Also presented in Fig. 2 is the mean-square displacement of the positrons as a function of time, $\left\langle z^{2}\right\rangle$. Two interesting features are immediately apparent. First, $\left\langle z^{2}\right\rangle$ becomes linear in the diffusion domain (i.e., $\left.t\right\rangle 1.0 \mathrm{ps}$ ), as expected. Inasmuch as the final broadening of the positron cloud can be described by a simple diffusion equation with a constant, thermal-energy diffusion coefficient, $D_{+}$, we expect the mean-square displacement to vary linearly over time, $\left\langle z^{2}\right\rangle \sim$ $D_{+} t$. This permits us to calculate the positron diffusion coefficient; in the case of $\mathrm{Al}$, we find $D_{+}=1.1(1) \mathrm{cm}^{2} / \mathrm{s}$, in good agreement with the experimental value of $D_{+}=1.7(2) \mathrm{cm}^{2} / \mathrm{s}$, obtained by H. Huomo et al.[11]

Second, the values of $\left\langle z^{2}\right\rangle$ at the point of crossover into the diffusion domain are not negligible. For Monte-Carlo simulations which terminate at higher energies (e.g., $25 \mathrm{eV}$ ), this distance represents the approximate length scale over which the neglect of the low-energy processes may become important. This has a direct bearing on the case of (narrow) stopping profiles for positrons incident at low kinetic energies; this is discussed in more detail below.

\section{STOPPING PROFILES AND MEAN DEPTH}

We next explore the effects of low-energy inelastic scattering on the positron stopping profiles and mean depths. To accomplish this, we adopt for initial conditions the fitted Makhovian distributions for $25 \mathrm{eV}$-endpoint profiles generated by BNLPOS and reported in Ref. 13. We use these fitted profiles for computational simplicity and due to the near-universal use of the Makhovian 
distribution to date; however the program is quite general and actual particle information (location and angular distribution) from any Monte-Carlo simulation can be used. We then continue the energy lose process from $25 \mathrm{eV}$ to 0.2 $\mathrm{eV}$, the approximate onset energy for positron diffusion, according to the results of the previous section.

The initial $25 \mathrm{eV}$ stopping profiles, together with the low-energy-broadened profiles, are reported in Fig. 3. Two qualitative festures are immediately apparent. First, there is clearly a non-vanishing opatial probability density for positrons at shallow depths. This is in stark contrast to the Makhovian distribution, for which $P(z=0)=0$. In cases in which other stopping profiles are taken for initial conditions, such as the results of Jensen, Walker, and Bouarissa (which predict a non-zero surface density of positrons at $100 \mathrm{eV}$ endpoint energies), we expect the effect of the low-energy processes will be to increase the density at the surface.[15]

Second, we observe that the greatest relative effect obtained by extending positron transport from $25 \mathrm{eV}$ to thermal energies occurs for positrons initially incident at low kinetic energies. Above $\sim 3 \mathrm{keV}$ incident energy, we see the low-energy scattering has little effect on the initial profiles. This is in agreement with the results of the previous section, in which we calculated the meansquare displacement of an initial delta-function distribution. The resulting lowenergy stopping profiles are, in a broad sense, a convolution of the initial profiles with the $100 \AA$-wide broadened delta-function distribution. For incident energies above $\sim 3 \mathrm{keV}$, the $25 \mathrm{eV}$ profiles in $\mathrm{Al}$ are sufficiently wide that the additional low-energy broadening has little relative effect.

To report these data quantitatively, we have attempted to fit the profiles with an analytical function. Neither the Makhovian distribution nor a recent modification of this provided an adequate fit. We did find, however, that the data were exceptionally well-described by the functional form proposed in the recent experimental study by Baker et al.[12],

$$
P(z)=-\frac{d}{d z} \exp \left\{-\left[\frac{z}{z_{0}}\left(1+\frac{z}{z_{0}}\right)^{2}\right]^{m}\right\}
$$

where we find $m$ (shape parameter) and $z_{0}$ (depth parameter) to be both material and energy dependent. In the case of $A l$, we find $<m>=1.15(1)$ and $\left\langle z_{0} / z_{1 / 2}\right\rangle=2.62(0)$. These values are in excellent agreement with the experimental values, but the importance of this agreement will be further discussed in Sec. VI. We also note this form adequately describes stopping profiles for systems with positive work functions, as discussed below.

The mean implantation depths are reported in Fig. 4. As can be seen, the effect of the low-energy scattering is to cause a deviation from the power-law form predicted by BNLPOS. In particular, there is a relative increase in the mean depth at low incident energies, resulting in the introduction of a slight positive curvature. This is in accord with the expected behavior: low-energy scattering results in a substatial egress of positrons from the solid in this case (zero work function), thereby depleting the overall concentration near the surface, 


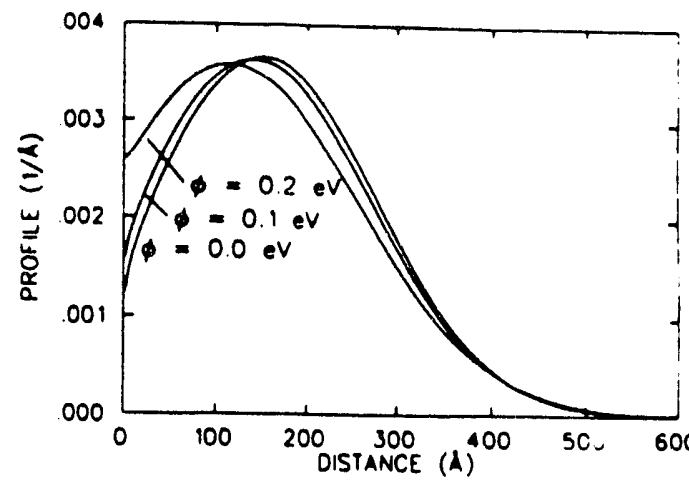

Pigure 3. Comparison of 25- $\mathrm{eV}$-endpoint profiles in $A$, and profiles for poritrons at thermal energy.

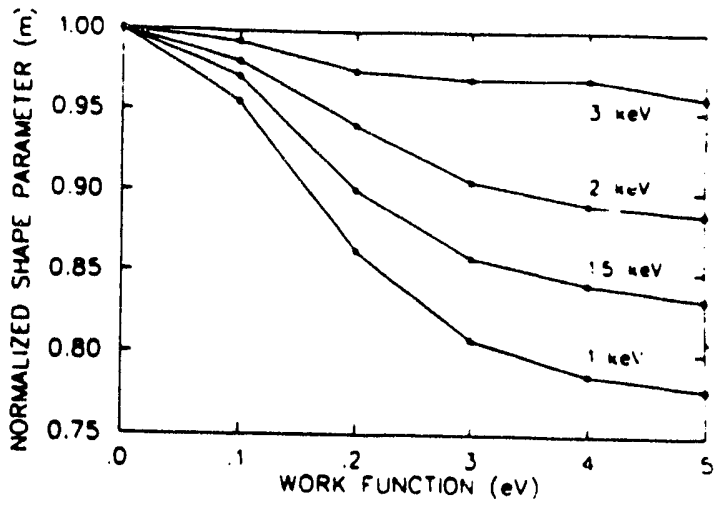

Figure 4. Comparion of mean depth for poaitrons at $25 \mathrm{eV}$ (dached line) and poxitrons at thermal energy.

and resulting in increased penetration and, therefore, increased mean depth. The positive curvature in the energy-dependence of the mean depth has been reported in a recent experimental study by Baker et al.[13]; we comment further on this in Sec. VI.

\section{WORK FUNCTION EFFECTS}

The continuation of the energy loss process to thermal energies permits us to examine, for the first time, the effect of a positron work function on the implantation profile. We consider the case of a positive work function $\phi_{+}$, and assume that this can be described by a square energy step barrier at the surface of height $\phi_{+}$. Recent theoretical work regarding positron-surface interactions suggests that this simple model may not be optimal, but it serves as a first step in examining work function effects.[14] As discussed earlier, positrons incident on this barrier (from within the solid) for which the kinetic energy $E_{\perp}$ of the component of velocity normal to the surface is less than the positron work function, $E_{\perp}<\phi_{+}$, are specularly reflected; for positrons with $E_{\perp}>\phi_{+}$, a small fraction is considered undergo quantum mechanical barrier reflection.

Stopping profiles for $1 \mathrm{keV}$ incidence energy in $\mathrm{Al}$ are presented in Fig. 5, for assumed work functions of $0 \mathrm{eV}$ though $0.2 \mathrm{eV}$. The number density of positrons near the surface increases as the magnitude of the work function, as expected: as the height of the energy step barrier increases, fe-er positrons are able to overcome this barrier and escape via reemission.

We have found these profiles to be well-described by Eq. (1). In Fig. 6, we present the dependance of the shape parameter $m$ on the assumed work function, obtained by fitting the stopping profiles to Eq. (1), for positrons incident at several kinetic energies on Al. Two qualitative features are immediately apparent. First, the greatest effect of the work function occurs for positrons incident at the lowest kinetic energies. In this case, the mean depth is small and the majority of positrons are within scattering distance of the surface. Second, as the work function increases, the resulting effect on the profiles dimishes and eventually 


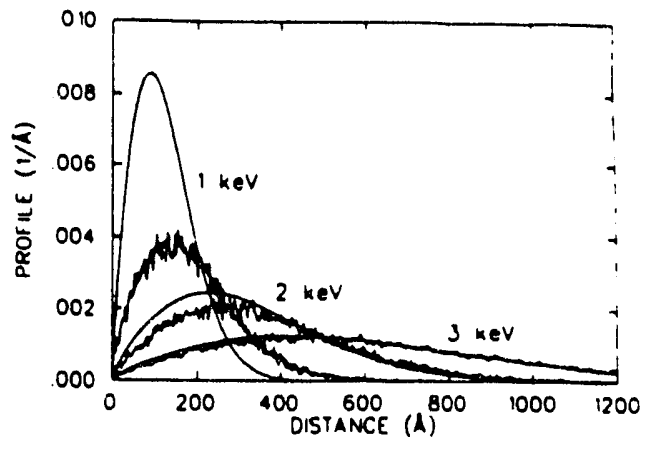

Figure 5. Stopping profiles for poxitrons incident at $1 \mathrm{keV}$ in $\mathrm{A}$, for different values of the positron work function.

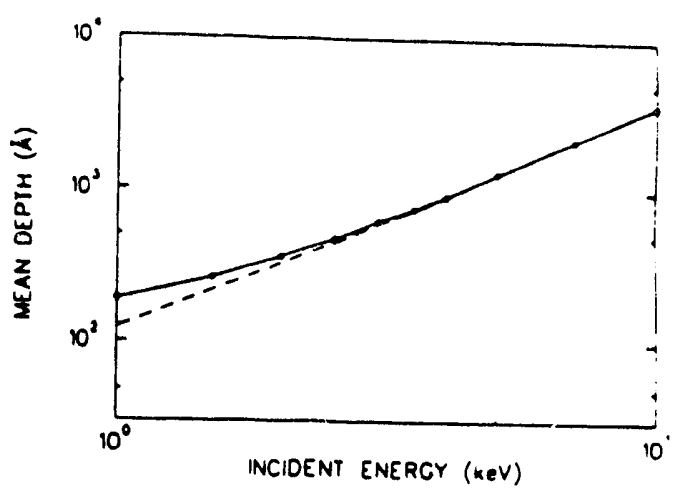

Figure 6. Values of the shape parameter $(m)$ for fits of Eq. (1) to the stopping profiles in $\mathrm{Al}$.

vanishes, as indicated by the levelling of the curves in the direction of increasing work function. As the size of the energy step barrier increases, few positrons are able to overcome this barrier and escape. Increasing the barrier further thus has little effect, as positrons unable to overcome a small barrier are certainly unable to overcome a larger one.

The ability to examine work function effects may have application to the study of multilayers and buried interfaces. It has already been shown by independent Monte-Carlo calculations that the high-endpoint-energy stopping profiles may change dramatically across an interface, particularly an interface between materials of different density. $[15,16]$ The effect of low-energy scattering will be to either increase or decrease the profile differences across the interface, depending on the gradient of the work function at the interface.

\section{SUMMARY AND DISCUSSION}

In the present work we have continued the positron energy loss process from $25 \mathrm{eV}$ (the usual cut-off for Monte-Carlo models of positron stopping) to nearthermal energies in $\mathrm{Al}$, via conduction-electron and acoustic phonon scattering. We have detailed the energy loss process, and we have determined a diffusion coefficient for $\mathrm{Al}$ which is in good agreement with experiment: We have shown that the final stages of thermalization can have important effects on the stopping profiles and mean depth, particulariy for positrons incident at low kinetic energies. We have also shown that a positive positron work function can have important effects on the stopping profiles. We have obtained agreement between our stopping profiles and those reported in the experimental study by Baker and co-workers. We have also shown that low-energy inelastic scattering can lead to a positive curvature in the incident-energy-dependence of the mean depth, also in good agreement with the experimental results of these authors.

It is the chief reward of theoretical physics to obtain agreement with experimental data. In the present work, we are not displeased with the agreement between our calculations and the recent experimental findings. In the case of 
Monte-Carlo simulations, however, particularly those in which there are many material-dependent variables to consider, agreement between theory and experiment is only a first step. The ultimate aim of this work is the construction of a model to provide accurate information about a wide variety of systems and which can be used in support of experimental data analysis or further theoretical studies.

For all who await this goal, there is an important point to consider. In every Monte-Carlo model of positron stopping constructed thus far, there are many adjustable parameters. In some cases, such as the present work, these adjustable parameters are obvious and take the form of phyaical quantities (such as the Debye temperature or the deformation potential), experimental measurements of which are not in full agreement with each other, or else are in disagreement with the results of theoretical calculations. In other cases, the adjustable parameters are far more subtle: for example, exactly how the electronic structure of atoms should be partitioned for elastic scattering calculations, or exactly when the optical mean free path model can be applied. Fortunately, the situation for positron Monte-Carlo models is in better shape than for many other models, and we need not contend with free parameters such as the inner-potentials or imaginary-potentials as are found in many coherent-wave calculations.

Our understanding of individual positron scattering mechanisms is probably sufficient to permit us to expect that accurate models of implantation can be constructed. Whether such models now exist, or whether more complicated models are necessary, such as those which can account for particle channeling at higher incident energies, is still a topic for research. Therefore, exhaustive benchmarking and comparisons between the results of Monte-Carlo calculations and experimental dsta must continue, so that a consistant set of material-dependent parameters can be attained; and so that, if necessary, the theoretical descriptions of positron-solid interactions or the Monte-Carlo models themselves can be refined.

\section{ACKNOWLEDGEMENTS}

It is a pleasure to thank J. Oliva, R. Nieminen, T. McMullen, and D. Britton for useful discussions. We gratefully thank the New Energy and Industrial Technology Development Organization (NEDO) for financial support, and DOE contracts DE-AC02-76CH00016, DE-FG02-91ER45439.

\section{REFERENCES}

1. Ritley, K. A., M. McKeown, and K .G. Lynn, Positron Beams for Solids and Surfaces, ed. by P. Schultz, G. R. Massoumi, P. J. Simpson, (American Institute of Physics, New York, 1990), pp. 3-18, and references therein.

2. Ghosh, V. J., Welch, D. O., and K. G. Lynn, pre-print.

3. Jensen, K., A. Walker, and N. Bouarissa, Positron Beams for Solids and Surfaces, ed. by P. Schultz, G. R. Massoumi, P. J. Simpson, (American Institute of Physics, New York, 1990), pp. 19-28. 
4. Valkealahti, S. and R. M. Nieminen, App. Phys. A 32, 95-106 (1983); and Valkealahti, S. and R. M. Nieminen, App. Phys. A 35, $51-59$ (1984).

5. Jensen, K. O., A. B. Walker, J. Phys. Condensed Matter 2, 9757-9775 (1990).

6. Jacoboni, C., Lugli, P., The Monte-Carlo method for semiconductior device simulation, (Springer-Verlag, Berlin, 1989).

7. Ritley, K. A., K. G. Lynn, V. J. Ghosh, D. O. Welch, preprint.

8. Asoka-Kumar, P., and K. G. Lynn, App. Phys. Lett. 57, 1634-1636 (1990).

9. Oliva, J., Phys. Rev. B 21, 4909 (1980).

10. Nieminen, R. M. , and J. Oliva, Phys. Rev. B 22, 2226-2247 (1980).

11. Huomo, H., E. Soininen, and A. Vehanen, App. Phys. A 49, 647-658 (1989).

12. Baker, J. A., N. B. Chilton, and P. G. Coleman, App. Phys. Lett. 59, 164-166 (1991).

13. Baker, J. A., N. B. Chilton, K. O. Jensen, A. B. Walker, and P. G. Coleman, J. Phys. Condensed Matt. 3, 4109-4114 (1991).

14. Kong, Y., K. G. Lynn Phys. Rev. 44, 13109-13111 (1991).

15. Ghosh, V. J., D. O. Welch, and K. G. Lynn, in these proceedings.

16. Aers, G. C., K. O. Jensen, A. B. Walker, "Calculation of positron diffusion in layered systems," SLOPOS5, August, 1992. 

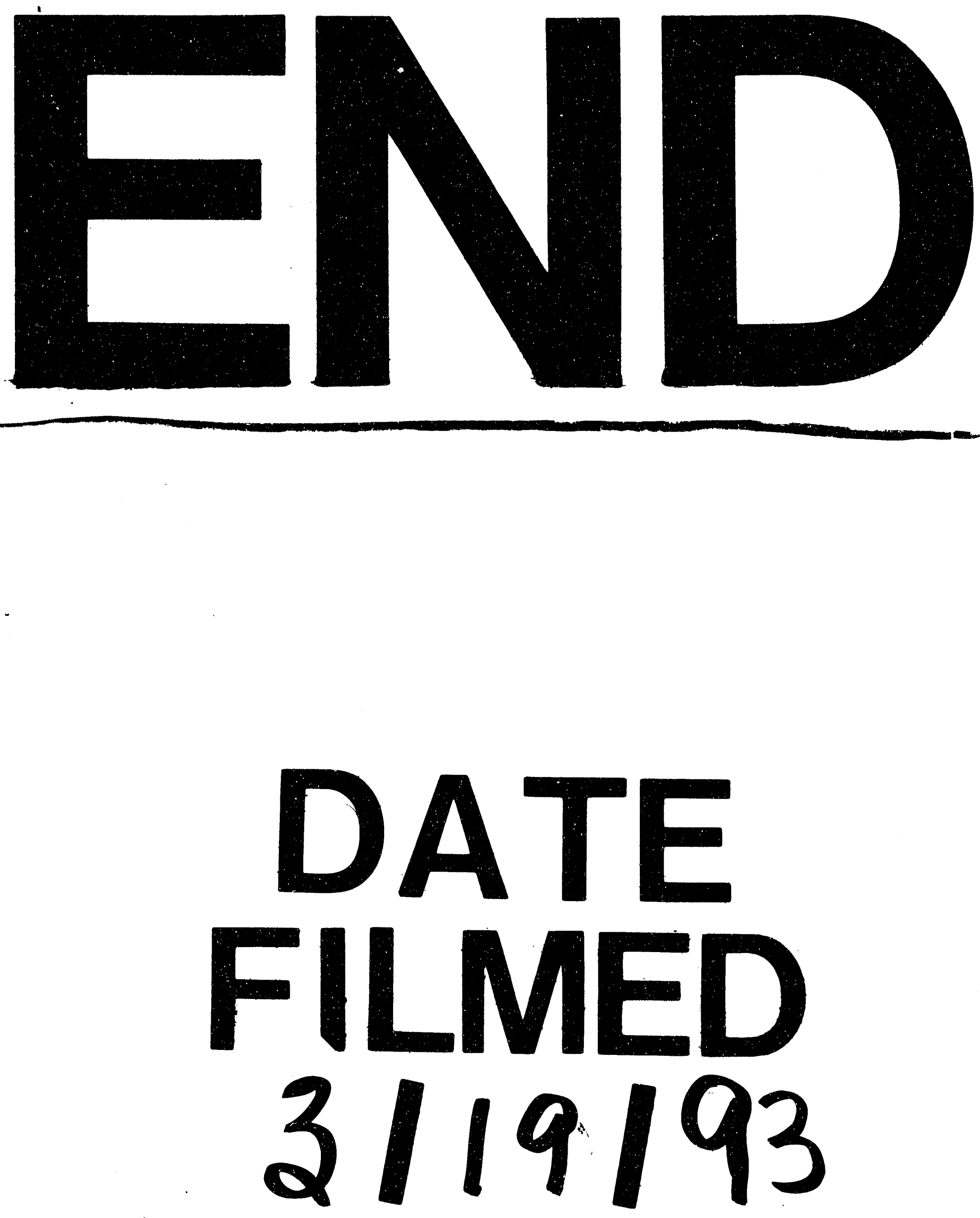


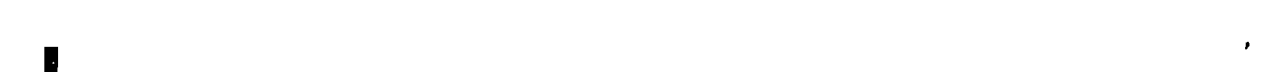

$-$ 